\title{
Modified Riemann-Liouville Approach to Field Theory and Particle Physics
}

\author{
J. Weberszpil, Cresus F. L. Godinho, \\ Instituto Multidisciplinar, Departamento de Tecnologia e Linguagens; Departamento de Física-UFRRJ \\ Av. Governador Roberto Silveira s/n- Nova Iguaçú, RJ e \\ BR 465-07, 23890-971, Seropédica, RJ \\ E-mail: josewebe@ufrrj.br E-mail: crgodinho@ufrrj.br
}

\section{J.A. Helayël-Neto \\ $\mathrm{CBPF}$}

Rua Dr Xavier Sigaud 150, 22290-180, RJ

E-mail: helayel@cbpf.br

\begin{abstract}
In this contribution we present our recent results where we have applied a modified form of Riemann-Liouville fractional derivative to build up a generalized fractional D'Alembertian and by means of a transformation of variables to light-cone coordinates, an explicit analytical solution was obtained. We have also set up the coarse-grained formulation of a fractional Schrödinger equation that incorporates a higher (spatial) derivative term which accounts for relativistic effects up to the lowest order in momentum.
\end{abstract}

Key-words: Fractional Calculus, Fractional Field Theory, Fractional Quantum Mechanics

\section{Introduction}

In recent works we have argued that the modeling of TeV-physics may demand an approach based on fractal operators and fractional calculus (FC) and we claimed that, in the realm of complexity, nonlocal theories and memory effects were connected to complexity and also that the FC and the nondifferentiable nature of the microscopic dynamics may be connected with time scales. Using the modified Riemann-Liouville (MRL) definition of fractional derivatives, we have worked out explicit solutions to a fractional wave equation (FWE) with suitable initial conditions to carefully understand the time evolution of classical fields with fractional dynamics. First, by considering space-time partial fractional derivatives of the same order in time and space, a generalized fractional D'Alembertian is introduced. By means of a transformation of variables to light-cone coordinates, an explicit analytical solution was obtained. Also, aspects connected with Lorentz symmetry were analyzed by using two different approaches.

To justify the coarse-grained approach we can see that it seems that a reasonable way to probe the classical framework of physics is to remark that, in the space of our real world, the generic point is not infinitely small (or thin), it rather has a finite size.

Also, we have pursued an investigation of the coarse-grained fractional Schrödinger equation (FSE), corrected by a fourth spatial derivative term which accounts for lowest power in momentum (relativistic) correction to the kinetic energy term. The corresponding continuity equation was worked out and we had also identified the contribution of the relativistic correction to the quantum potential in the coarse-grained treatment. As a consequence, in the classical regime, 
we derived the sort of fractional Newtonian law with the quantum potential included. The fractional counterparts of the De Broglie's energy and momentum relations were also presented and discussed. An attempt to formulate equations that describe dispersion relations for massless particles and refraction index for a coarse-grained medium was presented.

\section{Fractional D'Alembertian of same space-time partial deriva- tive order}

The D'Alembertian can now be established as:

$$
\frac{\partial^{\alpha}}{\partial x^{\alpha}} \frac{\partial^{\alpha}}{\partial x^{\alpha}} \phi(x, t)-\frac{1}{v^{2 \alpha}} \frac{\partial^{\alpha}}{\partial t^{\alpha}} \frac{\partial^{\alpha}}{\partial t^{\alpha}} \phi(x, t)=0, \quad 0<\alpha<1
$$

Proceeding now a variable change in a light cone, right and left movers, respectively, we can write $\xi=x-v t$ and $\eta=x+v t$.

Assuming a non-differentiable space of solutions, the chain rule in the MRL sense is[2] $\frac{d^{\alpha}}{d x^{\alpha}} f[u(x)]=\frac{d^{\alpha} f}{d u^{\alpha}}\left(\frac{d u}{d x}\right)^{\alpha}$. After some algebraic manipulations, we obtain $\frac{\partial^{\alpha}}{\partial \xi^{\alpha}} \frac{\partial^{\alpha}}{\partial \eta^{\alpha}} \phi(\xi, \eta)=0$.

\section{Solutions in the light-cone coordinates:}

The form of the Eq. (1) suggests a solution of the form, as in the case of integer derivatives: $\tilde{\phi}(\xi, \eta)=f(\xi)+g(\eta)$, subject to the initial conditions $\phi(x, 0)=F(x),\left.\frac{\partial^{\alpha} \phi(x, t)}{\partial t^{\alpha}}\right|_{t=0} \equiv G_{*}(x)$. According to the initial conditions, $F(x)=f(x)+g(x)$.

We have then found the functional forms of $f$ and $g$, so that the general solution for a general instant of time, $t$, can be expressed for $\phi(\xi, \eta)$. The expression is long and can be found in our reference[1].

The general result can indicate that a regularized definition could give a option to conserve the parity or the chiral properties of the field.

In the sequence we propose an alternative approach by considering fractional space-time instead of fractional space functions, that is, we consider that a coarse-grained space-time, which means that space and time are non-differentiable and considering the chain rule as [2] $\frac{d^{\alpha}}{d x^{\alpha}} f[u(x)]=\frac{d}{d u} f \frac{d^{\alpha}}{d x^{\alpha}} u$.

It can be shown that the ansatz $\phi=\phi\left(x^{\alpha}+\lambda t^{\beta}\right)$ is a solution of the FWE in a coarse-grained space-time [2], subject to the condition $\lambda_{\alpha, \beta}= \pm v^{\beta} \frac{\Gamma(\alpha+1)}{\Gamma(\beta+1)}$.

The result above gives the insight to redefine the light-cone variables $\xi, \eta$ as $\xi=x^{\alpha}-\lambda t^{\beta}$ and $\eta=x^{\alpha}+\lambda t^{\beta}$.

With the chain rule in the MRL sense, after some algebraic manipulations, we obtain a simple form $\frac{\partial^{2}}{\partial \xi \partial \eta} \phi(\xi, \eta)=0$, subject to the above conditions, permits to apply the same procedure used previously. The result is an expression for $\phi(\xi, \eta)[1]$.

The advantage of this approach, in a fractional coarse grained space-time, is that there is no violation of chirality and it opens the perspective to study higher orders derivatives in fractional space-time.

The introduction of higher derivatives yields the so-called negative squared-norm ghost states. Here, we argue that the presence of fractional higher derivatives might remove the problem of these unphysical modes.

\section{Lorentz transforms and invariance conditions}

Considering now the fractional front wave as

$$
c^{2 \alpha}\left(t^{\alpha}\right)^{2}-\left(x^{\alpha}\right)^{2}-\left(y^{\alpha}\right)^{2}-\left(z^{\alpha}\right)^{2}=c^{2 \alpha}\left(t^{\alpha}\right)^{2}=c^{2 \alpha}\left(t^{\prime \alpha}\right)^{2}-\left(x^{\prime \alpha}\right)^{2}-\left(y^{\prime \alpha}\right)^{2}-\left(z^{\prime \alpha}\right)^{2},
$$


we suppose a fractional transformation of form $x^{\prime \alpha}=\gamma_{\alpha, \beta}\left(x^{\alpha}-\lambda t^{\beta}\right)$ and $t^{\prime \beta}=\gamma_{\alpha, \beta}\left(t^{\beta}-\frac{\lambda}{c^{2 \beta}} x^{\alpha}\right)$.

The inverse transform can directly be obtained as $x^{\alpha}=\gamma_{\alpha, \beta}\left(x^{\prime \alpha}+\lambda t^{\prime \beta}\right), t^{\beta}=\gamma_{\alpha, \beta}\left(t^{\prime \beta}+\right.$ $\left.\frac{\lambda}{c^{2 \beta}} x^{\prime \alpha}\right)$.

From Eq. (2), with the transformations above, we are lead to a fractional gamma factor which reads as $\gamma_{\alpha, \beta}=\left(1-\frac{\lambda_{\alpha, \beta}^{2}}{c^{2 \beta}}\right)^{-1 / 2}$.

\section{Fractional Lorentz transform invariance for coarse-grained space-time}

We can show that a function of $\phi\left(x^{\alpha}, t^{\beta}\right)$ is a solution of the FWE. It can be shown that this wave equation is Invariant to Fractional Lorentz transform [1].

\section{Standard Lorentz invariance for non-differentiable space of solutions}

It can also be proved that in the space of non-differentiable solutions, the FWE is Lorentz invariant by standard Lorentz transforms, if exponents of fractional derivatives in space and time are equal to each other [1].

\section{An explicit example of solution}

As an illustrative example, let us take our initial conditions as $F(x)=\phi(x, 0)=\ominus(x) \ominus(1-x)$, where $\ominus(x)$ is the Heaviside function and, $\left.\frac{\partial^{\alpha} \phi(x, t)}{\partial t^{\alpha}}\right|_{t=0} \equiv G_{*}(x)=0$.

The solution obtained represents two well localized propagating rectangular pulses, propagating in opposite directions, with different attenuation parameters that depends on the chirality and the fractional exponent. Again, if the fractional exponent is one of those that preserves the chiral symmetry, the solution is identical to the case of an integer exponent.

For the case of fractional space-time, the solutions is similar for this example but with different space and time scales with the chiral symmetry preserved.

\section{The Fractional Klein Gordon Equation}

The fractional Klein Gordon equation (FKGE) can be written here as:

$$
\frac{1}{c^{2 \beta}} \frac{\partial^{2 \beta}}{\partial t^{2 \beta}} \psi(x, t)-M_{x, \alpha}^{2} \frac{\partial^{2 \alpha}}{\partial x^{2 \alpha}} \psi(x, t)+\frac{m^{2 \beta} c^{2 \beta}}{\hbar^{2 \beta}} \psi(x, t)=0 .
$$

The diffusion factor, $M_{x, \alpha}$, is here introduced for dimensional consistency reasons. This equation has also to be consistent with an fractional relativistic energy-momentum equation, given by $E^{\beta}=\sqrt{p^{2 \alpha} c^{2 \alpha}+m^{2 \beta} c^{4 \beta}}$.

\section{Fractional Schrödinger Equation with Lowest-Order Relativis- tic Correction}

Here we obtain the lowest-order relativistic correction to a FSE, with different orders for the fractional derivatives in time and space. This is carried out with the use of a FKGE.

We develop fractional relativistic energy-momentum equation in McLaurin's series by taking $f(x)=\left(1+x_{\alpha, \beta}\right)^{1 / 2}$ and assuming that $f^{(\alpha k)}(x)$ has a sequential character like $f^{(2 \alpha)}(x)=$ $\frac{\partial^{2 \alpha}}{\partial x^{2 \alpha}}=\frac{\partial^{\alpha}}{\partial x^{\alpha}} \frac{\partial^{\alpha}}{\partial x^{\alpha}}$.

Here, we propose the operators: $\widehat{E}^{\beta}=i(\hbar)^{\beta} \frac{\partial^{\beta}}{\partial t^{\beta}}$ and $\widehat{p}^{\alpha}=-i(\hbar)^{\alpha} M_{x, \alpha} \frac{\partial^{\alpha}}{\partial x^{\alpha}}$. 
After soma algebra, we are lead to one possible representation of the fractional Schrödinger equation given by

$$
i(\hbar)^{\beta} \frac{\partial^{\beta} \psi(x, t)}{\partial t^{\beta}}=-M_{x, \alpha}^{2} \frac{\hbar^{2 \alpha}}{2 m^{\beta}} \frac{c^{2 \alpha}}{c^{2 \beta}} \frac{\partial^{2 \alpha} \psi(x, t)}{\partial x^{2 \alpha}}+V_{\alpha, \beta} \psi(x, t)-\frac{1}{8} M_{x, \alpha}^{4} \frac{\hbar^{4 \alpha}}{m^{3 \beta}} \frac{c^{4 \alpha}}{c^{6 \beta}} \frac{\partial^{4 \alpha} \psi(x, t)}{\partial x^{4 \alpha}}
$$

\section{Fractional Continuity Equation}

We have found a fractional continuity equation, where the probability is defined as usually: $P=\psi^{*}(x, t) \psi(x, t)$.

After some algebra, we can be write $\frac{\partial^{\beta} \rho(x, t)}{\partial t^{\beta}}+\frac{\partial^{\alpha} J(x, t)}{\partial x^{\alpha}}=0$ where $\rho(x, t) \equiv \psi^{*}(x, t) \psi(x, t)$. The expression for $J$ can be found in ref. [3].

The above continuity equation shows that the probability is conserved in the fractional sense. Taking $\alpha=\beta=1$, we obtain the integer continuity equation with the lowest-order relativistic correction.

\section{Fractional Quantum Potential with Lowest-Order Relativistic Correction terms}

Now, we shall build up the fractional Bohmian equations, by parameterizing the solution of eq. (4) as $\Psi(\mathbf{r}, t)=R(\mathbf{r}, t) e^{i S(\mathbf{r}, t) / \hbar}$, where $R$ and $S$ are the amplitude of probability density and phase of $\Psi$, respectively, both being real-valued functions. Substituting this relation into the FSE and multiplying by $e^{-i S(\mathbf{r}, t) / \hbar}$, after some algebra and taking real and imaginary parts, we get two equations that lead to a fractional version of Bohmian Mechanics, including the its lowest-order relativistic correction limit. The fractional quantum potential, $Q^{\alpha}(x, t)$, was identified due to the presence of Planck constant. The long expression is omitted here.

With some more algebra we have also obtained the expression

$-\frac{\partial^{\alpha}}{\partial x^{\alpha}}\left(Q^{\alpha}(x, t)+V\right)=\frac{\partial^{\alpha}}{\partial x^{\alpha}}\left[M_{x, \alpha}^{2} \frac{\hbar^{2 \alpha}}{2 m^{\alpha}} \frac{1}{\hbar^{2}}\left(\frac{\partial^{\alpha} S}{\partial x^{\alpha}}\right)^{2}-\frac{1}{8} M_{x, \alpha}^{4} \frac{\hbar^{4 \alpha}}{m^{3 \alpha}} \frac{1}{c^{2 \alpha}} \frac{1}{\hbar^{4}}\left(S^{(\alpha)}\right)^{4}\right]+\hbar^{\alpha-1} \frac{\partial^{\alpha}}{\partial t^{\alpha}} \frac{\partial^{\alpha} S}{\partial x^{\alpha}}$.

Defining the fractional moment as $p^{\alpha}=M_{x, \alpha} \hbar^{\alpha-1} \frac{\partial^{\alpha} S}{\partial x^{\alpha}}$, and with a similar definition of the fractional velocity, that relates it to a fractional linear momentum, $v^{\alpha}=\left(\frac{d x}{d t}\right)^{\alpha}=\lambda_{\alpha, \beta} p^{\alpha}$, with $\lambda_{\alpha, \beta}=\left(M_{x, \alpha} \frac{c^{\alpha}}{c^{\beta}}\right)^{-1}$, we shall have that $-\frac{\partial^{\alpha}}{\partial x^{\alpha}}\left(Q^{\alpha}(x, t)+V\right) \equiv F^{\alpha}$, where $F^{\alpha}$ is defined as the fractional force. The equation above gives us a Newtonian-like fractional dynamical equation, that coincides with $\frac{d^{\alpha} p^{\alpha}}{d t^{\alpha}}$ if $\alpha=1$ and we do not consider the higher order term.

We define the fractional mechanical energy and the kinetic energy, respectively, as $E^{\alpha}(x, t)=$ $-\hbar^{\alpha-1} \frac{\partial^{\alpha} S(x, t)}{\partial t^{\alpha}}$, and $K^{\alpha}(x, t)=M_{x, \alpha}^{2} \frac{\hbar^{2 \alpha}}{2 m^{\alpha}} \frac{1}{\hbar^{2}}\left(\frac{\partial^{\alpha} S}{\partial x^{\alpha}}\right)^{2}-\frac{1}{8} M_{x, \alpha}^{4} \frac{\hbar^{4 \alpha}}{m^{3 \alpha}} \frac{1}{c^{2 \alpha}} \frac{1}{\hbar^{4}}\left(S^{(\alpha)}\right)^{4}$.

In terms of these and the quantum potential, we can rewrite $E^{\alpha}(x, t)=K^{\alpha}(x, t)+Q^{\alpha}(x, t)+$ $V$.

It is important to notice that, if we make $\alpha=1$, all the results are in complete agreement with standard Bohmian mechanics with the inclusion of lower relativistic correction terms.

The expressions for the fractional moment and the fractional energy open up the possibility for the attainment of anomalous dispersion relations.

Another point to highlight concerns energy conservation. If we assume for the phase $\mathrm{S}$ a dependence like a power of time, $S(x, t)=\hbar\left(f-\omega^{\alpha} t^{\alpha}\right)$, where $\omega_{\alpha}$ is a multiplicative constant and $f$ is some functions depending explicitly only on $x$, then we obtain for the fractional energy $E^{\alpha}(x, t)=-\hbar^{\alpha-1} \frac{\partial^{\alpha} S(x, t)}{\partial t^{\alpha}}=\hbar^{\alpha} \Gamma(\alpha+1) \omega^{\alpha}$, that is a constant. The fractional energy is conserved, provided an appropriate choice of phase is made. 


\section{De Broglie relations}

In what follows, we argue that fractional De Broglie relations in the coarse-grained context naturally emerge from the energy-phase and momentum-phase relationship .

If we write for the phase $\mathrm{S}$ a dependence like a power of time, $S(x, t)=\hbar\left[k^{\alpha} x^{\alpha} \pm \omega^{\alpha} t^{\alpha}\right]$, we have the fractional energy $E^{\alpha}(x, t)=-\hbar^{\alpha-1} \frac{\partial^{\alpha} S(x, t)}{\partial t^{\alpha}}=\hbar^{\alpha} \Gamma(\alpha+1) \omega^{\alpha}$, for the energy, which is a fractional Planck-type energy relation. This concept has already been proposed in the literature, in the context of soft matter. The expression above indicates that the Planck quantum energy relation may describe a fractional package of energy, changed from the usual one by the imposed fractionality of interactions and the medium. Notice that whenever $\alpha=1$, the usual quantum energy $E=\hbar \omega$ is recovered. Now, with the phase S into kinetic energy equation gives $K^{\alpha}(x, t)=M_{x, \alpha}^{2} \frac{1}{2 m^{\alpha}} \frac{1}{\hbar^{2}}\left[\Gamma(\alpha+1) \hbar^{\alpha} k^{\alpha}\right]^{2}+\mathcal{O}\left(p^{4}\right)$.

This suggests a definition of the momentum as $p^{\alpha}=M_{x, \alpha} \Gamma(\alpha+1) \hbar^{\alpha} k^{\alpha}$, which reduces to De Broglie relations of ordinary Quantum Mechanics for $\alpha=1$.

\section{Anomalous Dispersion Relation for Massless Particles}

Based on the fractional relativistic energy-momentum equation, with $m^{\alpha}=0$, the dispersion relation for a massless particle can be written as $E^{\alpha}=\sqrt{p^{2 \alpha} c^{2 \alpha}}$; it is a non-linear relation.

As an example, one can write[3] $\left[\hbar^{\alpha} \Gamma(\alpha+1)\right]^{2} \omega^{2 \alpha}=p^{2 \alpha} c^{2 \alpha}=M_{x, \alpha}^{2} \Gamma^{2}(\alpha+1) \hbar^{2 \alpha} k^{2 \alpha} c^{2 \alpha}$, or simplifying, the dispersion relation can be cast in terms in terms of $\omega^{\alpha}$ and $k^{\alpha}$ as $\omega^{\alpha}=M_{x, \alpha} k^{\alpha} c^{\alpha}$.

\section{Application: Group and Phase Velocities, The Dynamical Evolution Equation for the Phase and the Medium Refrac- tive Index}

As a general application, we develop expressions for group and phase velocities. Also, a simple dynamical evolution equation for the phase $S$ is obtained and a connection with refractive index of the medium is suggested.

The fractional group velocity can be written as $v_{g}^{\gamma}=\frac{d^{\gamma} E^{\alpha}}{\left(d p^{\alpha}\right)^{\gamma}}=\frac{\Gamma(\alpha+1)}{\Gamma(\alpha-\gamma+1)} p^{\alpha-\gamma} c^{\alpha}$, and the phase velocity can be expressed as the ratio between fractional energy and momentum is $v_{p h}=\frac{E^{\alpha}}{p^{\alpha}}=c^{\alpha}$.

Comparing both expressions, we can see that the phase velocity and the group velocity are equal only in the integer limit where $\alpha=\gamma=1$. Also, the group velocity has an non-linear behavior for non-integer fractionality. The expressions above indicate that the propagation of energetic particles in a coarse-grained medium might travel with a velocity $c^{\alpha}$, less than the speed of light $c$ in the trivial vacuum. We remark here that this was achieved without the necessity to modification of Maxwell's equations.

The the calculation of the group velocity is carried out with a different fractional order derivative parameter $\gamma$ (instead of $\alpha$ ). We argue that we may be eventually trying to describe the dynamics of a system with some slightly different fractional parameter. The fractionality of the medium may have changed slightly in some way, due to perturbation interactions. The changes, even if small, may give rise to the non-linear behavior described by the fractional expression obtained above.

With the help of the dispersion relation for a massless particle obtained, we can write an equation for the dynamical evolution of the phase $S$. Then, we write[3] $\frac{\partial^{\alpha}}{\partial t^{\alpha}} S(x, t)+M_{x, \alpha} \frac{\partial^{\alpha} S}{\partial x^{\alpha}} c^{\alpha}=$ 0 .

The canonical momentum-energy relation may be connected with the refractive index and can be written as a reference equation, relating the photon propagation velocity in the coarse-grained 
medium and the standard velocity of light in the trivial vacuum. This indicates an energydependent vacuum refractive index, $\frac{p^{\alpha}}{E^{\alpha}}=\frac{n_{\alpha}}{c}$, with $n_{\alpha}$ being the refractive index for a coarsegrained non-trivial optical medium. If the kinetic moments are considered, then $\frac{p^{\alpha}}{E^{\alpha}}=\frac{1}{n_{\alpha} c}$.

The expressions above, may provide a mechanism to attain a refraction index for the medium in a coarse-grained scenario and indicate there may be a close connection between fractionality and anomalous dispersion relations for photons, in a coarse-grained medium.

\section{Concluding Comments}

In this contribution, we relate our recent results. We have obtained a FWE in terms of a fractional D'Alembertian with a sequential form of MRL. With a particular choice of light-cone coordinates along with the use of sequential MRL derivative, leading to technique that creates a perspective to obtain solutions for other similar problems. Our solutions are worked out for general initial conditions. Since we are choosing to work with non-differentiable functions or a coarse-grained space-time, no use of distributional generalized functions or fractional powers of operators, neither the maintenance of semi-group properties of exponents in the derivatives is made. In each the cases of study the results agrees with standard integer order in the convenient limits.

Complementary, we have explicitly shown that the FWE in terms of the non-differentiable space-time is invariant under a Lorentz transform-like called fractional Lorentz transform, within the conditions of equality for fractional orders of derivatives in space and in time. Similar results from FWE in a non-differentiable space of solutions functions in terms of standard Lorentz transform.

We have worked out a suggested version of a fractional Schrödinger equation, with a lowestorder relativistic correction, obtained from a fractional wave equation [1] to which a mass term has been adjoined, to give us a FKGE. With the definition of some fractional operators, the McLaurin expansion and an ansatz for the plane wave solutions, we have obtained fractional versions of Bohmian equations to describe the particle dynamics associated with Bohmian mechanics, in the space of non-integer differentiable functions. We have also presented a formulation for an anomalous dispersion relation and to a refraction index, related to massless particle in a coarse-grained media and a vacuum refractive index for a coarse-grained non-trivial optical medium.

Also, a version of fractional De Broglie relations naturally comes out from our equations and we recover the integer relations in the convenient limit. In connection with the probability conservation, in the fractional case, we have worked out, to the lowest order in the relativistic correction, the fractional probability current. The probability can be conserved in this nondifferentiable space-time if we consider a fractional version of continuity equation that reduces to the standard one in the integer limit or, in other words, integer dimensions.

We have also proposed a formulation for an anomalous dispersion relation and a refraction index, related to massless particles in a coarse-grained medium and a vacuum refractive index for a coarse-grained non-trivial optical medium, that may indicates that energetic particles in a coarse-grained medium might travel with a velocity $c^{\alpha}$ less than the corresponding one in a non-interacting vacuum with speed of light $c$.

\section{References}

[1] Cresus F.L. Godinho, J. Weberszpil, J.A. Helayël-Neto, Extending the D'Alembert Solution to Space-Time Modified Riemann-Liouville Fractional Wave Equations, Chaos, Solitons \& Fractal, Chaos, Solitons \& Fractals, 45, 765-771 (2012). 
[2] Guy Jumarie, An approach to differential geometry of fractional order via modified RiemannLiouville derivative, Acta Mathematica Sinica, Published online: February 13, (2012).

[3] J. Weberszpil, C.F.L. Godinho, A. Cherman and J.A. Helayël-Neto, Aspects of the CoarseGrained-Based Approach to a Low-Relativistic Fractional Schrödinger Equation, In: 7th Conference Mathematical Methods in Physics - ICMP 2012, Rio de Janeiro. Proceedings of Science (PoS). Trieste, Italia: SISSA p. 1-19 (2012). 